\title{
An Outbreak of Crimean-Congo Hemorrhagic Fever in the South West of Iran
}

\author{
Mostafa Salehi-Vaziri, ${ }^{1}$ Shokrollah Salmanzadeh, ${ }^{2}$ Vahid Baniasadi, ${ }^{1}$ Tahmineh Jalali, ${ }^{1,3}$ Tahereh \\ Mohammadi, ${ }^{1}$ Sanam Azad-Manjiri, ${ }^{1}$ Yasaman Jamshidi, ${ }^{1}$ Sahar Khakifirouz,, and Mehdi Fazlalipour ${ }^{1,}$ \\ ${ }^{1}$ Department of Arboviruses and Viral Hemorrhagic Fevers (National Ref Lab), Pasteur Institute of Iran, Tehran, IR Iran \\ ${ }^{2}$ Health Research Institute, Infectious and Tropical Diseases Research Center, Faculty of Medicine, Ahvaz Jundishapur University of Medical Sciences, Ahvaz, IR Iran \\ ${ }^{3}$ Department of Biology, Shahed University, Tehran, IR Iran \\ "Corresponding author: Mehdi Fazlalipour, Department of Arboviruses and Viral Hemorrhagic Fevers (National Ref Lab), Pasteur Institute of Iran, 69 Pasteur Ave, Postcode: \\ 1316943551, Tehran, IR Iran. Tel/Fax: +98-2164112821, E-mail: mfp.virology@gmail.com, m_fazlalipour@pasteur.ac.ir
}

Received 2016 August 23; Revised 2016 November 15; Accepted 2016 December 03.

\begin{abstract}
Introduction: Crimean-Congo hemorrhagic fever (CCHF) is an acute viral zoonotic disease, which is endemic in vast geographic areas including the Middle East. The causative agent, Crimean-Congo hemorrhagic fever virus (CCHFV), is a Nairovirus, which is mainly transmitted to human from infected hard ticks and viremic livestock.

Case Presentation: In April 2016, an outbreak of CCHF occurred in Khuzestan province, Iran, because of slaughtering a tick-infested calf and manipulation of its meat.

Discussion: Given that viremic livestock are the main source of CCHF outbreaks in Iran, limitation of the livestock smuggling and unhealthy slaughtering is of great importance in the prevention of CCHF in endemic regions.
\end{abstract}

Keywords: Crimean-Congo Hemorrhagic Fever, Disease Outbreaks, Iran

\section{Introduction}

Crimean-Congo hemorrhagic fever virus (CCHFV) is an enveloped RNA virus, which is classified in Nairovirus genus, Bunyaviridae family (1). The virus is distributed throughout more than 30 countries in Africa, Asia, southeast Europe, and the Middle East including Iran (2). CCHFV is a life threatening tick-borne virus causing a fatal hemorrhagic fever, called CCHF, in human. The majority of CCHF cases are sporadic; however, outbreaks have been reported in some regions (3). CCHFV can be transmitted to people through the bite of infected Ixodid (hard) ticks and direct exposure to body fluids or tissues from viremic livestock or other patients $(4,5)$. Therefore, the outbreaks often involve people working in livestock industry and health care workers. Here, we report a CCHF outbreak in Khuzestan province, southwest of Iran, caused by direct contact with infected blood or tissues of a calf and consumption of the under-cooked infected meat.

\section{Outbreak Presentation}

In April 2016, three patients presenting clinical pictures compatible with CCHF were admitted to a regional hospital in Khuzestan province (Figure 1).

Case 1 was a 34 year-old slaughterer man who referred to the hospital with sudden fever $\left(>38^{\circ} \mathrm{C}\right)$, petechiae, and

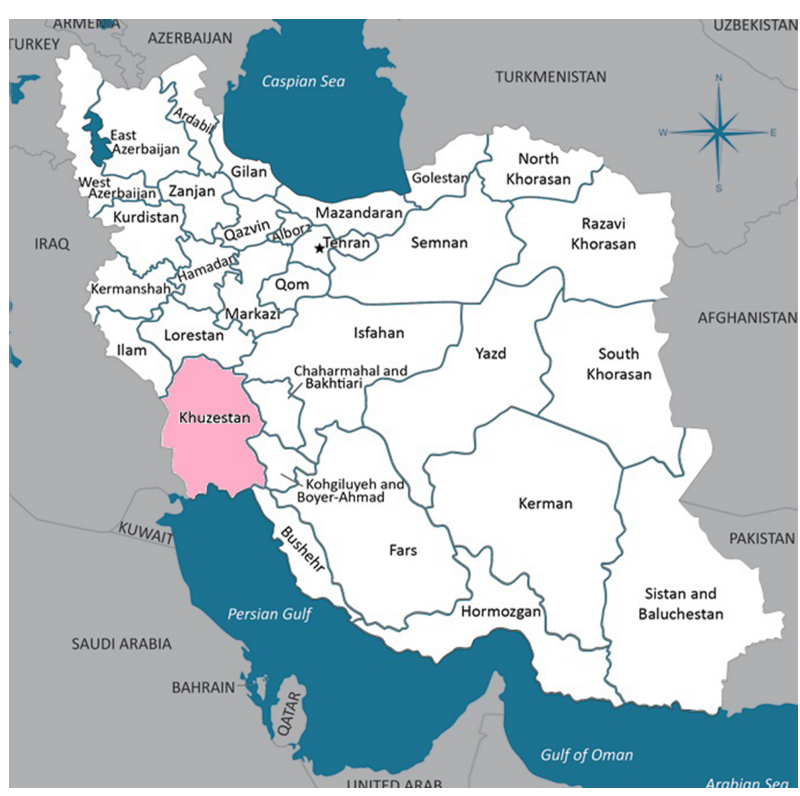

Figure 1. Geographical Location of Khuzestan Province in Iran

hemoptysis. Following laboratory analyses, severe thrombocytopenia, Leukocytosis, and elevated liver enzymes were seen. Splenomegaly was observed within ultrasonog- 
raphy. This case slaughtered a tick-infested calf within 3 days before onset of symptoms.

Case 2 was admitted with sudden fever, severe headache, myalgia, and hematemesis. Laboratory analyses showed thrombocytopenia and increased liver enzymes. This patient was a 17 year-old butcher man with no history of tick bit; he was chopping the calf meat that was slaughtered by case 1 .

Case 3 was a 27 year-old farmer who collaborated with case 2 in manipulation of the calf meat that was slaughtered by case 1 . He also consumed the meat of freshly slaughtered calf as an under-cooked kebab. The farmer was admitted to hospital with sudden onset of fever (> $38^{\circ} \mathrm{C}$ ), myalgia, nausea and vomiting, diarrhea, stomach ache, and epistaxis. More investigation revealed leukopenia, thrombocytopenia, and increased liver enzymes.

For all three cases, human immunodeficiency virus (HIV), hepatitis B virus (HBV), Hepatitis C virus (HCV), Malaria, Brucellosis, Leptospirosis, and salmonella tests were negative. All the patients were treated with Ribavirin and finally recovered. Moreover, their families were alerted for CCHF infection but they did not receive Ribavirin as a prophylaxis.

According to the Iranian national expert committee on viral hemorrhagic Fevers guideline, all CCHF suspected samples were referred to the department of arboviruses and viral hemorrhagic fevers, Pasteur Institute of Iran (National. Ref. Lab). To confirm CCHFV infection, the samples were analyzed by reverse transcription polymerase chain reaction (RT-PCR) and ELISA tests. Viral RNA was extracted using QIAamp RNA Easy Mini kit (QIAgen, GmbH, Hilden, Germany) according to the manufacturer's protocol, and RT-PCR was performed using Iran F2 and Iran R3 primers as previously described (6). Anti-CCHFV IgM was investigated using IgM capture ELISA (7).

All 3 cases tested positive for CCHFV RNA and antiCCHFV IgM. To ensure the same origin of infection, RT-PCR products were sent to Macrogen Company (Macrogen Inc., Seoul, Republic of Korea) for sequencing. The results of sequencing were confirmed by BLAST (http://blast.ncbi.nlm.nih.gov/Blast.cgi) and the sequences were submitted to GenBank under the accession numbers KX432283-5. Clustal W alignment showed that all 3 sequences were identical (Table 1 ).

\section{Discussion}

Ixodid ticks are reservoirs of CCHFV in nature while different animals including livestock are amplifying hosts for the virus $(1,4)$. CCHFV replication in amplifying hosts causes an asymptomatic infection that is undetectable (8, 9). Therefore, individuals working in the livestock industry
Table 1. Laboratory Findings of CCHF Patients on Admission

\begin{tabular}{lccc}
\hline Parameters & Case 1 & Case 2 & Case 3 \\
\hline AST, IU/L & 738 & 130 & 650 \\
ALT, IU/L & 401 & 249 & 335 \\
$\mathbf{A L P}, \mathbf{I U} / \mathbf{L}$ & 419 & 249 & 308 \\
$\mathbf{W B C}, \times \mathbf{1 0 0 0} / \mu \mathbf{L}$ & 12.2 & 5.3 & 2.8 \\
\hline Platelet, $\times \mathbf{1 0 0 0} / \mu \mathbf{L}$ & 24 & 45 & 12 \\
\hline
\end{tabular}

Abbreviations: ALP, alkaline phosphatase; ALT, alanine aminotransferase; AST, aspartate aminotransferase; WBC, white blood cells.

such as shepherds, abattoir workers, and butchers living in endemic areas are at occupational risk for CCHFV infection. Corresponding with this notion, most of CCHF cases in Iran have occurred among persons involving in this industry (10). Direct contact with blood and tissue of viremic livestock is a well-documented route of CCHFV transmission. In addition, there is some evidence that virus transmission can occur via consumption of raw or under-cooked infected meat $(6,11)$. In this outbreak, the exposure to tissue and blood of a viremic livestok was the main risk factor and also with respect to the case 3 , the consumption of raw meat could not be ruled out as a possible route of virus transmission.

In this outbreak, the time of interval between onset of symptoms and hospital admission was 3 days and therefore, all three cases recieved Ribavirin therapy at the earliest possible time, suggesting that fast monitoring and efficient surveillance system is very critical to reduce the burden of CCHF in endemic areas.

In conclusion, to control CCHF in Iran, people living in endemic areas, especially high risk groups, must be informed about routes of transmission and prevention of CCHF. Moreover, regular inspections from veterinary organization to limit the livestock smuggling, reduction of traditional or unhealthy slaughtering, and rapid treatment of tick-infested livestock could lead to a sharp reduction in the incidence rate of CCHF in Iran.

\section{Acknowledgments}

The authors acknowledge their gratitude to all the colleagues of Department of Arboviruses and Viral Hemorrhagic Fevers (National. Ref. Lab), Pasteur Institute of Iran, Tehran, Iran.

\section{Footnotes}

Authors' Contribution: All authors participated in the research design and contributed to different parts of the 
research.

Financial Disclosure: The authors have no relevant financial relationships to disclose for this article.

\section{References}

1. Shayan S, Bokaean M, Shahrivar MR, Chinikar S. CrimeanCongo Hemorrhagic Fever. Lab Med. 2015;46(3):180-9. doi: 10.1309/LMN1P2FRZ7BKZSCO. [PubMed: 26199256].

2. Bente DA, Forrester NL, Watts DM, McAuley AJ, Whitehouse CA, Bray M. Crimean-Congo hemorrhagic fever: history, epidemiology, pathogenesis, clinical syndrome and genetic diversity. Antiviral Res. 2013;100(1):159-89. doi: 10.1016/j.antiviral.2013.07.006. [PubMed: 23906741].

3. Aradaib IE, Erickson BR, Mustafa ME, Khristova ML, Saeed NS, Elageb $\mathrm{RM}$, et al. Nosocomial outbreak of Crimean-Congo hemorrhagic fever, Sudan. Emerg Infect Dis. 2010;16(5):837-9. doi: 10.3201/eid1605.091815. [PubMed: 20409377].

4. Panayotova E, Papa A, Trifonova I, Christova I. Crimean-Congo hemorrhagic fever virus lineages Europe 1 and Europe 2 in Bulgarian ticks. Ticks Tick Borne Dis. 2016;7(5):1024-8. doi: 10.1016/j.ttbdis.2016.05.010. [PubMed: 27378409].

5. Naderi HR, Sheybani F, Bojdi A, Khosravi N, Mostafavi I. Fatal nosocomial spread of Crimean-Congo hemorrhagic fever with very short incubation period. Am J Trop Med Hyg. 2013;88(3):469-71. doi: 10.4269/ajtmh.2012.12-0337. [PubMed: 23269658].

6. Fazlalipour M, Baniasadi V, Mirghiasi SM, Jalali T, Khakifirouz S, AzadManjiri S, et al. Crimean-Congo Hemorrhagic Fever Due to Consumption of Raw Meat: Case Reports From East-North of Iran. Jpn J Infect Dis. 2016;69(3):270-1. doi: 10.7883/yoken.JJID.2015.498. [PubMed: 26743144].

7. Chinikar S, Shayesteh M, Khakifirouz S, Jalali T, Rasi Varaie FS, Rafigh $\mathrm{M}$, et al. Nosocomial infection of Crimean-Congo haemorrhagic fever in eastern Iran: case report. Travel Med Infect Dis. 2013;11(4):252-5. doi: 10.1016/j.tmaid.2012.11.009. [PubMed: 23266037].

8. Akinci E, Bodur H, Leblebicioglu H. Pathogenesis of Crimean-Congo hemorrhagic fever. Vector Borne Zoonotic Dis. 2013;13(7):429-37. doi: 10.1089/vbz.2012.1061. [PubMed: 23663164].

9. Oncu S. Crimean-Congo hemorrhagic fever: an overview. Virol Sin. 2013;28(4):193-201. doi: 10.1007/s12250-013-3327-4. [PubMed: 23913177].

10. Chinikar S, Moghadam AH, Parizadeh SJ, Moradi M, Bayat N, Zeinali $M$, et al. Seroepidemiology of crimean congo hemorrhagic Fever in slaughterhouse workers in north eastern iran. Iran J Public Health. 2012;41(11):72-7. [PubMed: 23304679].

11. Sharifi-Mood B, Metanat M, Hashemi-Shahri SM, Mardani M, Hashemi SA, Fayyaz-Jahani F. Crimean-Congo hemorrhagic fever following consumption of uncooked liver: case series study. Iran J Clin Infect Dis. 2011;6(3):128-30. 